\title{
Nível de atividade física em trabalhadores das fábricas de Ubá-MG
}

\section{Physical activity level in workers of factories of Ubá-MC}

Como citar este artigo: BAIÃO, BRAÚlO P.; OLIVEIRA,

RENATA A. R.; OLIVEIRA, PEDRO V. S. R. MARINS, JOÃOC. B.

Nivel de atividade física em trabalhadores das fábricas de Ubá-MC. Revista Saúde (Sta. Maria). 2020; 46 (2)

\section{Autor correspondente:}

Nome: Braúlio Parma Baião

E-mail: braulio_parma@hotmail.com

Telefone: (32) 98407-4849

Formação Profissional: Graduando em Educação Física pelo Centro Universitário Governador Ozanam Coelho (UNIFACOC), Ubá, MG, Brasil.

Filiação Institucional: Centro Universitário Governador Ozanam Coelho

Endereço para correspondência: Rua: Euzira Teixeira Guimaraes

Bairro: Paulino Fernandes

Cidade: Ubá

Estado: Minas Gerais

CEP: 36503-022

Data de Submissão:

15/07/2020

Data de aceite:

07/10/2020

Conflito de Interesse: Não há conflito de interesse

\section{(cc) $\mathrm{BY}-\mathrm{NC}-\mathrm{ND}$}

\section{RESUMO}

Objetivo: Avaliar o nível de AF diário/semanal em trabalhadores de uma cidade no interior de Minas Gerais, e relacionar com parâmetros antropométricos e pressóricos. Metodologia: Realizou-se um estudo observacional com um delineamento transversal, entre março e agosto de 2019, com 128 trabalhadores do polo moveleiro de Ubá-MG. Foi aplicado o Questionário Internacional de Atividade Física (IPAQ), em sua versão curta. E realizada a avaliação antropométrica (índice de massa corporal (IMC), relação cintura quadril e percentual de gordura) e pressórica dos avaliados. Resultados: Pelo IPAQ, em torno de $60 \%$ dos avaliados apresentaram nível de AF satisfatório, Ativo ou Muito Ativo. $O$ restante dos avaliados foi, em maioria, classificado como sedentário (17,2\%). A pressão arterial sistólica (PAS) e a pressão arterial diastólica (PAD) apresentaram diferença entre os grupos de ativos/muito ativos [PAS: 120,00 (100-180) mmHg; PAD: $80,00(60-120) \mathrm{mmHg}$ ] e insuficiente ativos/sedentários [PAS: 120,00 (90-180) mmHg; PAD: 80,00 (60-120) mmHg]. Porém, o nível de AF não se associou com o excesso de peso $\left(\mathrm{IMC}>25 \mathrm{Kg} / \mathrm{m}^{2}\right)$ e hipertensão arterial. Conclusão: Os trabalhadores apresentaram um número regular de insuficientemente ativos e sedentários, porém este não se relacionou com as variáveis antropométricas e pressóricas.

PALAVRAS-CHAVE: Fatores de risco cardiovascular; Atividade física; Trabalhadores.

\section{ABSTRACT}

Objective: To evaluate the level of daily/weekly PA in workers from a city in the interior of Minas Gerais, and to relate it to anthropometric and pressure parameters. Methodology: An observational cross-sectional study was conducted between March and August 2019, with 128 workers from the furniture pole of Ubá-MG. The International Physical Activity Questionnaire (IPAQ) was applied in its short version. The anthropometric (body mass index (BMI), waist-to-hip ratio and fat percentage) and pressure evaluation of the evaluated subjects was performed. Results: By IPAQ, around $60 \%$ of the evaluated individuals presented satisfactory PA level, Active or Very Active. Most of the others were classified as sedentary (17.2\%). Systolic blood pressure (SBP) and diastolic blood pressure (DBP) showed a difference between the active / very active groups [SBP: 120,00 (100-180) mmHg; PAD: $80,00(60-120) \mathrm{mmHg}$ ] and insufficient. active/sedentary [PAS: 120,00 (90-180) mmHg; PAD: 80,00 $(60-120) \mathrm{mmHg}$. However, the level of PA was not associated with overweight $\left(\mathrm{BMl}>25 \mathrm{Kg} / \mathrm{m}^{2}\right)$ and hypertension. Conclusion: The workers had a regular number of insufficiently active and sedentary, but this was not related to the anthropometric and pressure variables.

KEYWORDS: Cardiovascular risk factors; Physical activity; Workers. 


\section{INTRODUÇÃO}

As doenças cardiovasculares (DCV) são as principais causas de óbito mundial, em que no Brasil representam $30 \%$ do total de mortes ${ }^{1}$. Vários fatores comportamentais estão associados a tais doenças, como o consumo de álcool e tabaco, a alimentação inadequada e a inatividade física. Dados obtidos pelo Vigitel de 2018, verificaram que dentre 27 capitais brasileiras, a frequência de adultos que não alcançaram um nível suficiente de prática de AF foi de $44,1 \%$, sendo 43,2\% em Belo Horizonte-MG².

A atividade física (AF) por sua vez é um importante meio de prevenir as $D C V$, visto que sua prática regular tem trazido vários benefícios à saúde. Porém, a falta dela ou de forma insuficiente também é preocupante. A urbanização, a industrialização e a violência urbana, são obstáculos para a prática regular de AF. Dessa forma, manter uma prática regular de exercícios físicos é cada vez mais importante para manter o nível AF recomendado³.

Um quadro de inatividade física está relacionado com o risco de mortalidade global. Assim que, de acordo com a American College of Sports Medicine (ACSM), um período de 30 minutos diários de AF com intensidade moderada cinco vezes na semana; ou 20 minutos de AF com intensidade vigorosa três vezes por semana já seria o suficiente para diminuir os riscos à saúde ${ }^{4}$.

A AF no lazer tem relação direta na melhoria da saúde, porém em relação a AF ocupacional os resultados são contraditórios, pois apesar de vários estudos demonstrarem benefícios da atividade laboral, sobre os fatores de risco cardiovascular e síndrome metabólica ${ }^{5,6}$; estudo recente realizado por Holtermann et al. ${ }^{7}$ apresentou que a AF no trabalho pode não fornecer benefícios para a saúde cardiovascular, podendo ainda prejudicar a saúde do trabalhador. Assim, vê-se a necessidade de avaliar o nível de AF e saúde cardiovascular dos trabalhadores de diferentes funções laborais, a fim de verificar seus possíveis benefícios.

Existem diversos estudos atuais sobre prevalência de fatores de risco cardiovascular em trabalhadores, como trabalhadores rurais, que encontrou relação de indicadores antropométricos com o Escore de Framingham ${ }^{8}$; trabalhadores bancários, que apresentaram elevados níveis de inatividade física, dislipidemias e excesso de peso9; professores de nível superior e educação básica ${ }^{10,11}$, que encontrou que a inatividade física está associada a um perfil de risco à saúde, e uma elevada prevalência de sobrepeso/obesidade, respectivamente; e trabalhadores da saúde ${ }^{12}$, que encontraram elevada prevalência de síndrome metabólica. Assim que, tais estudos demonstram um elevado fator de risco entre diferentes grupos ocupacionais, destacando a importância de uma intervenção nesse grupo.

Dessa forma, vê-se necessário averiguar o nível de AF de trabalhadores, em especial que atuam em fábricas, pois além da promoção a saúde, a prática de AF é um importante aliado da saúde pública. Sendo uma estratégia interessante, visto que os custos com o sistema de saúde e previdência social tem aumentado, devida à invalidez precoce e mortalidade da população. Além disso, sabe-se que a AF realizada de forma regular pode contribuir para redução da pressão arterial e composição corporal ${ }^{13}$, o qual é um dado importante, visto que tais fatores de risco apresentam associação com as DCV. 
Diante disso, o presente estudo tem como objetivo avaliar o nível de AF diário/semanal em trabalhadores de uma cidade no interior de Minas Gerais, e relacionar com parâmetros antropométricos e pressóricos.

\section{MÉTODOS}

\section{Caracterização da Pesquisa e Amostra}

Foi realizado um estudo observacional com um delineamento transversal, com 128 trabalhadores do polo moveleiro da cidade de Ubá-MG, com idade de 19 a 70 anos. A coleta de dados foi realizada no período de Março a Agosto de 2019.

O cálculo da amostra foi realizado no Programa Epi Info, versão 7.2.1.0. Considerando-se um tamanho da população infinita; com uma frequência esperada de 13,7\%, que foi estimado segundo os dados de inatividade física da população de Belo Horizonte-MG, Brasil2; com um erro padrão de $5 \%$ e um intervalo de confiança de $90 \%$, obteve-se 0 valor de no mínimo 126 trabalhadores necessários para o estudo.

Inicialmente os indivíduos foram orientados sobre os procedimentos de coleta de dados e assinaram o termo de consentimento livre e esclarecido. Todo o procedimento foi aprovado pelo comitê de ética com seres humanos da Universidade Federal de Viçosa (CAAE 87704818,8,0000,5153), seguindo a Resolução número 446/12 do Conselho Nacional de Saúde.

Foi realizado inicialmente o contato com 12 fábricas de móveis através de e-mail e telefone, em que foi apresentada a dinâmica do estudo, os procedimentos e objetivos da pesquisa. Para as fábricas interessadas foi informado aos trabalhadores sobre todos os procedimentos da pesquisa. Além disso, foi afixado cartazes com informações pontuais, no mural de aviso das fábricas e divulgado a pesquisa através dos meios virtuais (site e facebook).

Os indivíduos que concordaram em participar passaram por um processo de enquadramento nos critérios de inclusão. Foram considerados como critério de inclusão: ter pelo menos dois anos de atuação laboral; não possuir licença medica superior a uma semana no último ano, ou de dois meses nos últimos três anos; não estar em período gestacional; e não apresentar comprometimento orgânico e/ou metabólico que impeçam de participar do estudo. E como critério de exclusão: foi excluído os indivíduos que não responderam adequadamente todo o questionário de AF.

\section{Variáveis e Procedimentos de Coletas de Dados}

A obtenção dos dados foi realizada em seis fabricas em uma sala reservada e no laboratório de medidas antropométricas do Centro Universitário Governador Ozanam Coelho, em horário conforme disponibilidade do avaliado. Para a coleta os voluntários informaram seus dados pessoais como idade, sexo, seção em que trabalham e tempo de 
atuação. Nesse momento foi assinado o termo de consentimento livre e esclarecido, além da aplicação de questionário e realização das medidas antropométricas.

Para mensurar o nível de AF foi utilizada a versão curta do Questionário Internacional de Atividade Física (IPAQ) ${ }^{14}$, validado para população brasileira ${ }^{15}$ e já empregado em outros estudos ${ }^{10,16,17}$. Este instrumento contém perguntas relacionadas com as atividades realizadas em uma semana habitual, e as relaciona com as atividades realizadas no trabalho, lazer, deslocamento, em casa, e o tempo gasto em atividades na posição sentado. Tal questionário estima o tempo gasto em atividades físicas de intensidade moderada, vigorosa e caminhada. O IPAQ foi aplicado por três avaliadores capacitados e preparados para esclarecer qualquer dúvida dos participantes. Sendo posteriormente os indivíduos classificados de acordo com as recomendações do próprio instrumento em cinco categorias (Sedentário, Insuficientemente ativo A e B, Ativo e Muito ativo).

Para caracterização da amostra as medidas antropométricas foram realizadas por três profissionais qualificados, em uma sala reservada e os avaliados utilizando roupas leves e descalços. A estatura foi aferida em um estadiômetro portátil da marca WCS® (Cardiomed, Brasil), com precisão de $1 \mathrm{~mm}$ e capacidade de $210 \mathrm{~cm}$. A massa corporal do avaliado foi obtida em balança portátil da marca Plenna® (modelo Acqua SIM09190, Plena, Brasil), com capacidade para $150 \mathrm{~kg}$ e precisão de 100 gramas. $O$ índice de massa corporal (IMC) foi calculado utilizando a formula IMC= Massa corporal $(\mathrm{kg}) /$ Estatura $^{2}(\mathrm{~m})$, e classificado conforme recomendação da World Health Organization ${ }^{18}$.

As circunferências de cintura, abdômen e quadril foram aferidas utilizando uma fita antropométrica da marca Cescor $\circledast$, com metragem para $2 \mathrm{~m}$. A circunferência de cintura foi realizada na menor curvatura localizada entre as costelas e a crista ilíaca; a circunferência de quadril no ponto de maior protuberância; e a circunferência abdominal foi obtida na altura da cicatriz umbilical. A relação cintura quadril $(R C Q)$ foi calculada utilizando a seguinte formula $R C Q=$ circunferência de cintura $(\mathrm{cm}) /$ circunferência de quadril $(\mathrm{cm})$.

O cálculo do percentual de gordura corporal foi obtido por medida de dobras cutâneas. A técnica de coleta e a análise seguiram as recomendações dos protocolos de Jackson e Pollock ${ }^{19}$ e Jackson, Pollock e Ward²0, com aferição em três dobras (Mulheres: Tríceps, Supra ilíaca e Coxa; Homens; Peitoral, Abdômen e Coxa), medida com o adipômetro modelo Cescorf®. Foram realizadas três medidas alternada e utilizado o valor médio entre elas. Posteriormente foi feita a conversão da densidade corporal em percentual de gordura, através da fórmula de Siri21: $(\% G=[(4,95 / D C)-4,50]$ x 100).

O procedimento de verificação da pressão arterial foi realizado com o paciente sentado, com os pés apoiados no chão e separados na largura dos ombros, após um período de repouso de 5 minutos. 0 estetoscópio e esfigmomanômetro utilizado foi o aneróide da marca Premium $®$ (Wenzhou Instrument Co., China, 2014), todos devidamente calibrados e com braçadeira padrão para adultos. As medidas e critérios de classificação da pressão arterial foram realizadas de acordo com a Sociedade Brasileira de Cardiologia22. 


\section{Procedimentos Estatísticos}

Todos os dados foram submetidos ao teste de Komolgorov-Smirnov para verificar a pressuposição de normalidade em todas as variáveis, sendo que a massa corporal, estatura, circunferência de cintura e percentual de gordura apresentaram dados normais. A análise dos dados constituiu na exploração descritiva das variáveis estudadas (média e desvio-padrão para os dados paramétricos, e mediana e valores máximo e mínimo para os dados não paramétricos) e no cálculo das prevalências. O teste t Student foi utilizado para comparação entre os grupos, e seu correspondente (Mann-Whitney) para os dados não-paramétricos.

Para verificar as associações entre as variáveis categóricas foi usado o teste do qui-quadrado. A razão de chances (Odds Ratio) foi utilizada para determinar a força de associação entre as variáveis, com intervalo de confiança de $95 \%$. Para todos os tratamentos adotou-se um nível de significância menor que 5\%. Todas as análises estatísticas foram realizadas através do programa SPSS para Windows, versão 20.0 (Chicago, EUA).

\section{RESULTADOS}

A amostra final do estudo correspondeu a 128 trabalhadores, sendo 106 (82,8\%) do setor de produção e 22 $(17,2 \%)$ do setor administrativo. Do total, 94 eram do sexo masculino $(73,4 \%)$ e 34 do sexo feminino (26,6\%). A figura 1 apresenta o nível de AF dos trabalhadores.

Figura 1 - Classificação do Nível de Atividade dos trabalhadores, de acordo com o IPAQ, Ubá-MG, Brasil, 2019.

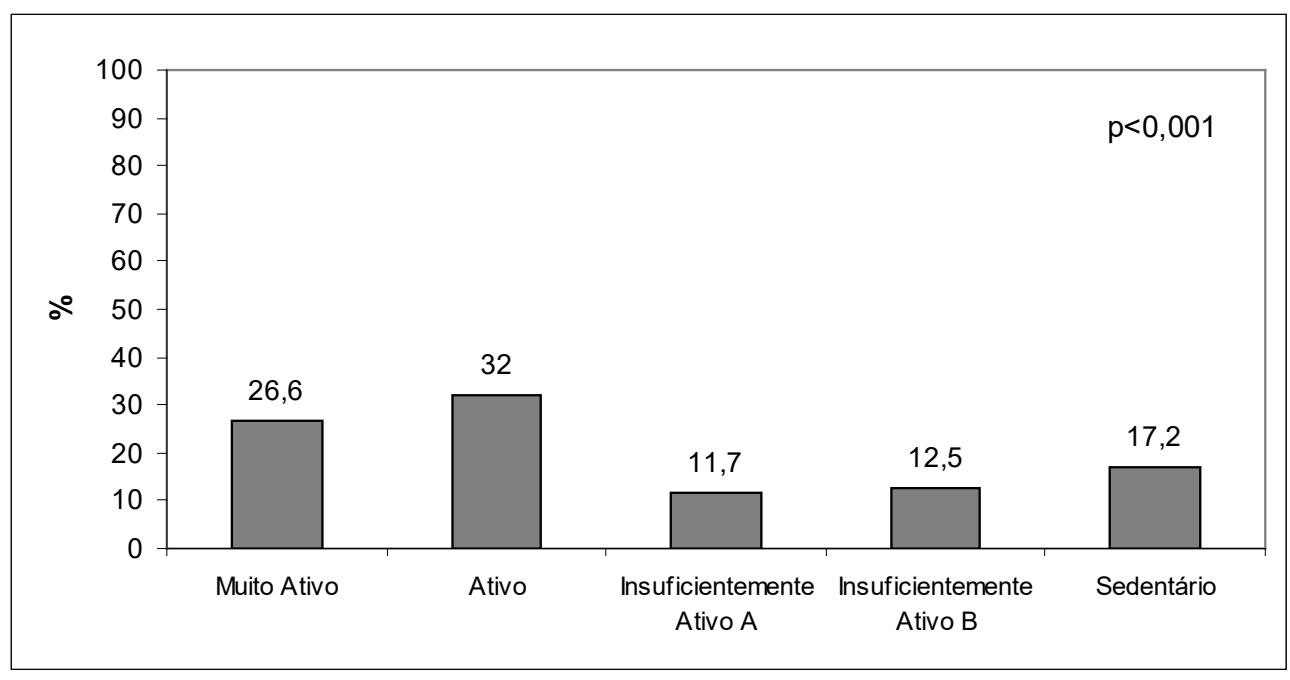

Fonte: Dados da Pesquisa. $p<0,05$ (teste qui quadrado). 
As medidas antropométricas e pressóricas, estratificadas segundo o nível de AF, estão apresentadas na tabela 1. A PAS e PAD foram as variáveis que obtiveram diferença estatisticamente significante entre os grupos $(p<0,05)$, porém sem significância clínica.

Tabela 1 - Características da amostra segundo a classificação do nível de atividade física, Ubá-MG, Brasil.

\begin{tabular}{llll}
\hline Variáveis & Ativo e & Insuficiente Ativo & P-valor \\
& Muito Ativo & e Sedentário & \\
& $(\mathbf{n}=75)$ & $(\mathbf{n}=53)$ & \\
\hline Idade (anos) & $34(19-70)$ & $33(19-64)$ & $0,324^{*}$ \\
Tempo de atuação (anos) & $15(1-53)$ & $12(1-43)$ & $0,688^{*}$ \\
Massa corporal (kg) & $74,27 \pm 14,08$ & $71,49 \pm 15,28$ & $0,291^{\neq}$ \\
Estatura (m) & $1,69 \pm 0,08$ & $1,67 \pm 0,08$ & $0,109^{\neq}$ \\
IMC (Kg/m²) & $26,00(18-49)$ & $23,70(18-51)$ & $0,472^{*}$ \\
CC (cm) & $84,22 \pm 10,87$ & $82,89 \pm 12,18$ & $0,521^{\neq}$ \\
CA (cm) & $87,00(67-139)$ & $83,50(66-142)$ & $0,272^{*}$ \\
CQ (cm) & $98,00(83-138)$ & $96,50(81-145)$ & $0,364^{*}$ \\
RCQ & $0,85 \pm 0,07$ & $0,84 \pm 0,09$ & $0,741^{\neq}$ \\
$\% G C$ & $22,21 \pm 9,64$ & $22,39 \pm 10,27$ & $0,919^{\neq}$ \\
PAS (mmHg) & $120,00(100-180)$ & $120,00(90-180)$ & $0,014^{*}$ \\
PAD (mmHg) & $80,00(60-120)$ & $80,00(60-120)$ & $0,031^{*}$ \\
\hline Font & & &
\end{tabular}

Fonte: Dados da Pesquisa.

* Dados são apresentados como mediana, e valores mínimo e máximo. Teste Mann-Whitney.

${ }^{*}$ Dados são apresentados como média e desvio-padrão. Teste t Student.

IMC: índice de massa corporal; CC: circunferência de cintura; CA: circunferência do abdômen; $C Q$ : circunferência do quadril; RCQ: relação cintura-quadril; \%GC: percentual de gordura coroporal; PAS: pressão arterial sistólica; PAD: pressão arterial diastólica.

Quando analisada a variação da distribuição dos dados da PAS e PAD, é possível observar que entre o grupo de ativo e muito ativo os dados apresentam-se menos dispersos (figura 2). 
Figura 2 - Histograma da PAS e PAD segundo a classificação do nível de atividade física, Ubá-MG, Brasil, 2019.

a) Ativo e Muito Ativo
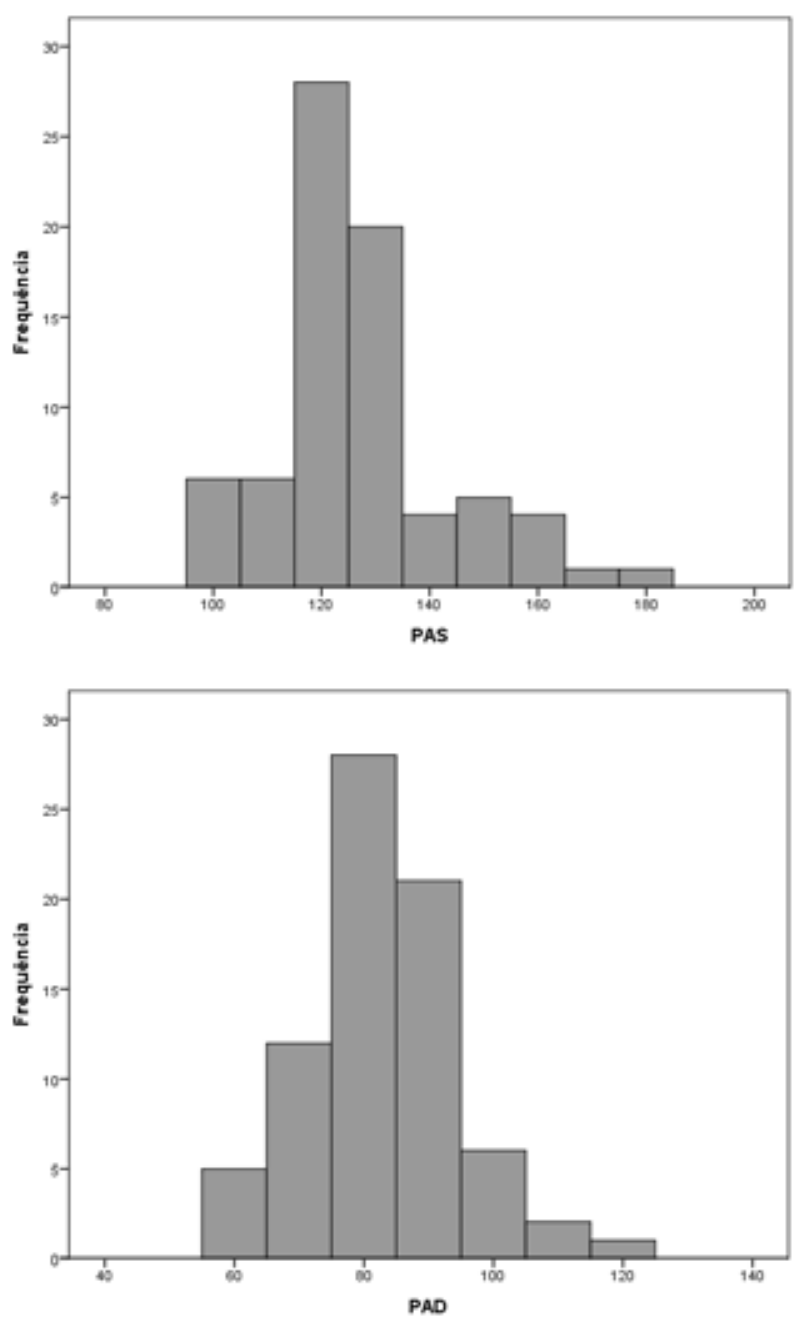

b) Insuficientemente Ativo e Sedentário
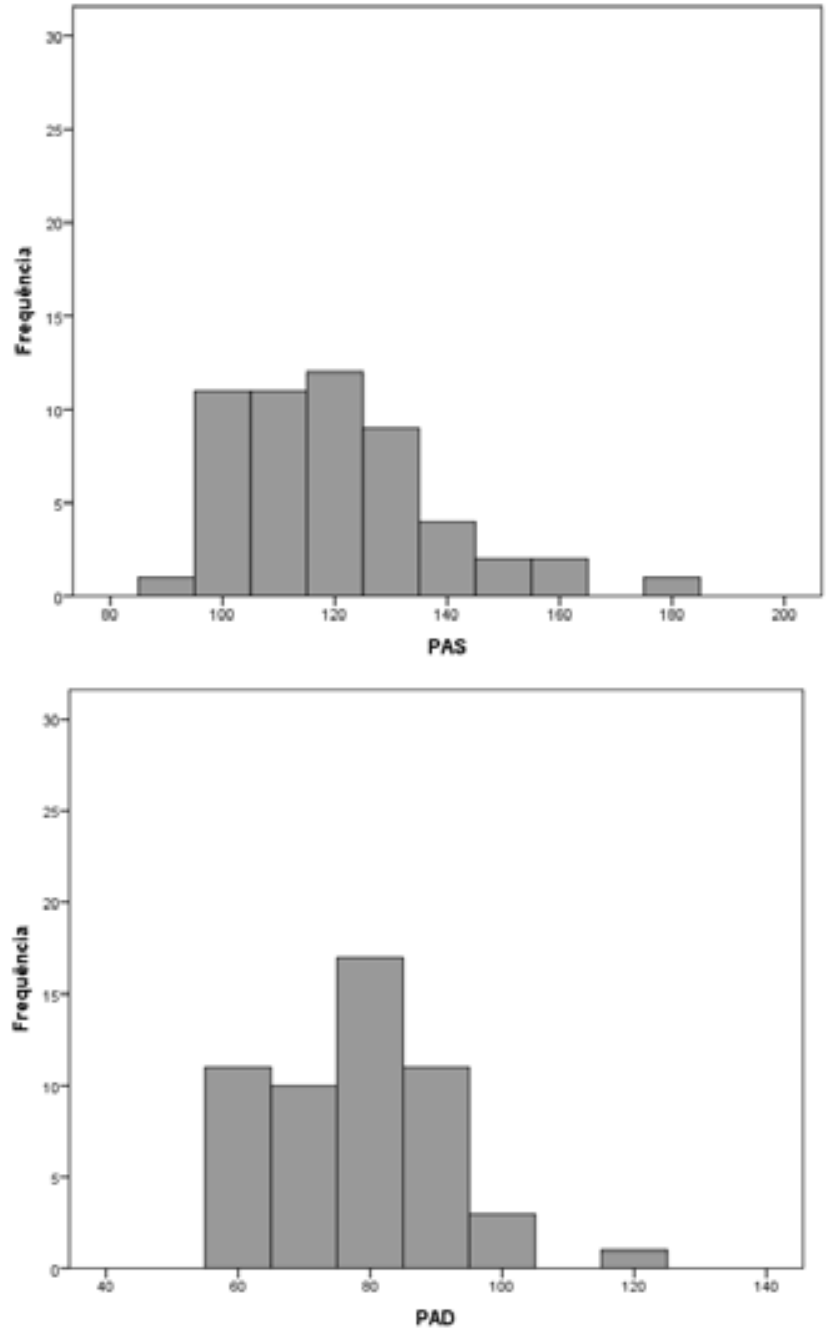

Fonte: Dados da Pesquisa.

PAS: pressão arterial sistólica; PAD: pressão arterial diastólica.

Na tabela 2 encontra-se os fatores de risco associados ao nível insuficiente de AF. Não foi possível observar associações entre as variáveis.

Tabela 2 - Análise dos fatores de risco cardiovascular associados ao nível insuficiente de atividade física, Ubá-MG, Brasil, 2019.

\begin{tabular}{clc}
\hline & $\begin{array}{l}\text { Razão de Chance } \\
(\text { IC95\%) }\end{array}$ & P-valor* $^{*}$ \\
\hline Excesso de Peso $^{1}$ & 0,540 & \\
& $(0,265-1,100)$ & 0,127 \\
Hipertensão Arterial $^{2}$ & 0,560 & \\
& $(0,264-1,191)$ & 0,185 \\
\hline
\end{tabular}

Fonte: Dados da Pesquisa.

* Teste do qui-quadrado de Pearson com correção de continuidade.

${ }^{1}$ Excesso de Peso: IMC > 25 Kg/m² 18; 2 Hipertensão Arterial: PAS > 140 mmHg e/ou PAD > 90 mmHg ${ }^{22}$. 


\section{DISCUSSÃO}

No presente estudo foi verificado, utilizando o IPAQ, que 41,4\% dos avaliados apresentaram nível insuficiente de AF (insuficientemente ativos e sedentários). Valores semelhantes foram encontrados pelo Ministério da Saúde, em que a frequência de adultos brasileiros com nível insuficiente de prática de AF alcançou 44,1\%2. Isto reforça que mesmo em uma cidade do interior de Minas Gerais, o estado de nível insuficiente de AF é elevado.

Quando analisado com dados também do IPAQ, percebe-se que o presente estudo apresentou resultados superiores ao do estudo de Mota Júnior et al. ${ }^{17}$, com professores da educação básica e ao estudo de Santana e Peixoto ${ }^{10}$, com professores universitários. Porém, estudo com servidores públicos, realizado por Polisseni e Ribeiro ${ }^{3}$ e estudo com bancários, realizado por Souza Júnior et al. ${ }^{16}$, no qual utilizaram IPAQ em sua versão curta, apresentou que $56,1 \%$ e $66,7 \%$ respectivamente, dos avaliados são inativos ou insuficientemente ativos, número superior ao presente estudo. Uma possível explicação pode ser devido à atividade laboral dos trabalhadores, pois bancários tendem a permanecer um elevado tempo na posição sentada. Além disso, destaca-se que no presente estudo $82,8 \%$ dos trabalhadores eram do setor de produção, o qual tendem a permanecer longas horas na posição em pé.

Porém, ao se comparar os dados do presente estudo com trabalhos realizados em trabalhadores que utilizaram o pedômetro como forma de medida, verifica-se que o percentual encontrado é inferior ao obtido com professores da educação básica ${ }^{23}$.

Ressalta-se que a aplicação de questionários subjetivos, como o IPAQ em pesquisas, apresentam pontos positivos e negativos. Dentre os positivos, pode-se destacar a fácil aplicabilidade e o baixo custo, obtendo assim a abrangência de uma grande amostra. No entanto, este instrumento tem apresentado baixa concordância quando comparado com instrumentos objetivos, tendendo a superestimar o nível de $\mathrm{AF}^{17}$.

A PAS e PAD apresentaram diferença estatística entre os grupos de ativos/muito ativos e insuficiente ativos/ sedentários $(p<0,05)$, porém sem significância clínica. Além disso, o nível de AF não se associou com a hipertensão arterial. Mas, sabe-se que o nível de AF se associa com os níveis pressóricos, conforme observado em trabalhadores dos setores de limpeza, transporte e manufatura ${ }^{24}$.

Também não foi possível observar associação entre o nível de AF e o excesso de peso. Porém, estudo com professores da educação básica e trabalhadores de empresa siderúrgica encontrou associação entre o nível de atividade e o excesso de peso ${ }^{11}$. De fato sabe-se que quanto mais inativo o sujeito é maior a chance do mesmo apresentar sobrepeso e consequentemente outras patologias como, por exemplo, a hipertensão arterial. Assim que, os resultados do presente estudo com ausência de associação surpreendem.

Está bem estabelecido que o excesso de gordura traz prejuízos à saúde, como a hipertensão arterial ${ }^{27}$ e diabetes mellitus $^{28}$. A AF aumenta o gasto calórico promovendo um aumento no metabolismo basal, contribuindo assim para a 
perda de peso e diminuição do risco de desenvolver outras doenças como a hipertensão e a obesidade. Dessa forma, mesmo no grupo avaliado não havendo relação entre AF e excesso de peso, destaca-se a necessidade de mudanças de hábitos entre este grupo de trabalhadores, como o aumento do nível de AF. Pois, estudo realizado por Oliveira et al. ${ }^{29}$ verificou que professores inativos apresentaram quatro vezes mais chances de desenvolver síndrome metabólica em comparação com os ativos.

Além disso, o valor mediano de percentual de gordura de $22 \%$ se mostra elevado, se levarmos em consideração que a maioria dos avaliados são do sexo masculino, com mediana de idade próxima aos 30 anos. Dessa forma, fica clara a necessidade de intervenção no grupo de trabalhadores avaliados.

O presente estudo apresentou algumas limitações, como a realização de um estudo transversal, o qual possibilita a ocorrência de causalidade reversa, que pode interferir na interpretação dos resultados. Pois, não se pode afirmar se o nível de AF tem impacto sobre os fatores de risco analisados; ou se os fatores de risco têm influência sobre o nível de AF dos trabalhadores. Segundo a utilização do IPAQ para avaliar o nível de AF, o que apenas permitiu uma avaliação subjetiva de tal variável.

Deve-se enfatizar a mudança do estilo de vida dos trabalhadores, através da prática regular de AF e boa alimentação. Uma estratégia interessante para a promoção da saúde destes, pode incluir a implementação de ginástica laboral para os mesmos.

\section{CONSIDERAÇÕES FINAIS}

Houve um número regular de trabalhadores insuficientemente ativos e sedentários, porém o nível de AF não se associou com as variáveis antropométricas e pressóricas dos avaliados.

\section{AGRADECIMENTOS}

Ao Centro Universitário Governador Ozanam Coelho pela bolsa de iniciação científica. 


\section{REFERÊNCIAS}

1. Rocha RM, Martins WA. Manual de prevenção cardiovascular. 1 ed. Rio de Janeiro: SOCERJ; 2017.

2. Brasil. Ministério da Saúde. Secretaria de Vigilância em Saúde. Secretaria de Gestão Estratégica e Participativa. Vigitel Brasil 2018: Vigilância de fatores de risco e proteção para doenças crônicas por inquérito telefônico. Brasília: Ministério da Saúde; 2019.

3. Polisseni MLC, Ribeiro LC. Exercise as protective fator for health of public servants. Rev Bras Med Esporte. $2014 ; 20(5): 340-4$.

4. Garber CE, Blissmer B, Deschenes MR, Franklin BA, Lamonte MJ, Lee IM, et al. Quantity and quality of exerxise for developing and maintaining cardiorespiratory, musculoskeletal, and neuromotor fitness in apparently healthy adults: guidance for prescribingexercise. Med Sci Sports Exerc. 2011; 43(7)1334-59.

5. Straub M, Foshag P, Przysylek B, Horlitz M, Lucia A, Sanchisgomar F, et al. Occupation and metabolic syndrome: is there corretation? A cross sectional study in different work activity occupations of German firefighters and office workers. Diabetol Metab Syndr. 2016; 8(1).

6. Kolchraiber FC, Rocha JS, César DJ, Monteiro OO, Frederico GA, Gamba MA. Level of physical activity in peoples with type-2 diabetes mellitus. Revista Cuidarte. 2018; 9(2):2105-16.

7. Holtermann A, Krause N, Beek AJVD, Straker L. The pysical activity paradox: six reasons why occupational physical activity (OPA) does not confer the cardiovascular health benefits that leisure time physical activity does. $\mathrm{Br} \mathrm{J}$ Sports Med. 2017; 52(3):149-50.

8. Pohl HH, Arnold EF, Dummel KL, Cerentini TM, Reuter EM, Reckziegel MB. Indicadores antropométricos e fatores de risco associados cardiovascular em trabalhadores rurais. Rev Bras Med Esporte 2018; 24(1):64-8.

9. Pertali GB, Salaroli LB, Bissoli NS, Zandonade E. Autoavaliação do estado de saúde e fatores associados: um estudo em trabalhadores bancários. Cad Saúde Pública. 2015; 31(4):787-99. 
10. Santana JO, Peixoto SV. Inatividade física e comportamentos adversos para a saúde entre professores universitários. Rev Bras Med Esporte. 2017; 23(2):103-8.

11. Oliveira RAR, Mota Júnior RJ, Tavares DDF, Moreira OC, Lima LM, Amorim PRS, et al. Prevalence of obesity and association of body mass index with risk factors in public school teachers. Rev Bras Cineantropom Desempenho Hum 2015; 17(6):742-52.

12. Ribeiro RP, Marziale MHP, Martins JT, Ribeiro PHV, Robazzi MLCC, Dalmas JC. Prevalência de síndrome metabólica entre trabalhadores de enfermagem e associação com estresse ocupacional, ansiedade e depressão. Rev Latino-Am Enfermagem. 2015; 23(3)435-40.

13. Rocha RM, Martins WA. Manual de prevenção cardiovascular. 1 ed. Rio de Janeiro: SOCERJ; 2017. Disponível em: https://socerj.org.br/wp-content/uploads//2017/05/Manual_de_Prevencao_Cardiovascular_SOCERJ.pdf. Acesso em: 23 de setembro de 2017.

14. International Physical Activity Questionnaire. Scoring Protocol; 2007. Disponível em: <https://sites.google.com/site/theipaq/>. Acesso em: 16 de Maio de 2019.

15. Matsudo S, Araújo T, Matsudo V, Andrade E, Oliveira LC, Braggion G. Questionário Internacional de Atividade Física (IPAQ): Estudo de validade e reprodutibilidade no Brasil. Rev Bras Ativ Fis Saúde 2001; 6(2).

16. Souza Júnior EV, Cunha CV, Oliveira BG, Bomfim ES, Boery RNSO, Boery EN. Level of physical activity and quality of life of bank workers. Enferm Actual Costa Rica. 2019; 36:36-47.

17. Mota Júnior RJ, Tavares DDF, Gomes AKV, Oliveira RAR, Marins JCB. Level of physical activity in basic education teachers evaluated by two instruments. Journal of Physical Education. 2017; 28:e2833.

18. World Health Organization. National Institutes of Health. Clinical Guidelines on the identification, evaluation, and treatment of overweight and obesity in adults-the evidence report. National Institutes of Health. Obesity Research 1998; 6:51-209. 
19. Jackson AS, Pollock ML. Generalized equations for predicting body density of men. Br J Nutr 1978;40:497-504.

20. Jackson AS, Pollock ML, Ward A. Generalized equations for predicting body density of women. Med Sci Sports Exerc 1980;12(3):175-81.

21. Siri WE. Body composition from fluid paces and density: analysis of methods. In: Brozek J, Henschel A. Techniques for measuring body composition. Washington: National Academy of Science; 1961.

22. Sociedade Brasileira de Cardiologia. VII Diretrizes Brasileiras de hipertensão. Arq Bras Cardiol 2016;107(3).

23. Oliveira RAR, Amorim PRS, Mota Júnior RJ, Tavares DDF, Faria FR, Moreira OC, et al. Association between the number of daily steps and the cardiovascular risk factors in basic education teachers. J Sports Med Phys Fitness. 2018; 58(5): 714-20.

24. Korshoj M, Clays E, Krause N, Gupta N, Jorgensen MB, Holtermann A. Associations between occupational relative aerobic workload and resting blood pressure among different age groups: a cross-sectional analysis in the DPhacto study. BMJ Open. 2019; 9(9): e029713.

25. Sabbahi A, Arena R, Elokda A, Phillipes SA. Exercise and hypertension: Uncovering the mechanisms of vascular control. Prog Cardiovasc Dis. 2016; 59(3):226-34.

26. Leggio M, Fusco A, Limongelli G, Sgorbini L. Exercise training in patients with pulmonary and systemic hypertension: A unique therapy for two different diseases. Eur J Intern Med. 2018; 47:17-24.

27. Oliveira RAR, Mota Júnior RJ, Tavares DDF, Moreira OC, Marins JCB. Fatores associados à pressão arterial elevada em professores da educação básica. Rev Educ Fís. 2015; 26(1):119-129.

28. Oliveira RAR, Amorim PRS, Moreira OC, Amorim W, Costa EG, Marins JCB. Relação de indicadores antropométricos com glicemia entre servidores universitários. Revista de Ciências Médica. 2015; 24(1):19-28.

29. Oliveira RAR, Amorim PRS, Moreira OC, Mota Júnior RJ, Lima LM, Marins JCB. Prevalence of Metabolic Syndrome and Associated Factors in Basic Education Teachers. Rev Andal Med Deporte. 2019; 12(3):216-20. 